\title{
Laser als Ersatz für Säge und Schere in der Unfallchirurgie? Eine vergleichende experimentelle Studie
}

\author{
M. Dressel, R. Jahn, H.U. Langendorf, W. Neu und K.H. Jungbluth
}

Laser-Laboratorium Göttingen e.V., Im Hassel 21, W-3400 Göttingen, Bundesrepublik Deutschland

Die Entwickung des Excimerlasers und dessen athermischer Abtragungsvorgang (Photoablation) eröffnen viele Möglichkeiten des Einsatzes in dem Gebiet der Unfallchirurgie, seitdem es mit Hilfe eines speziellen Einkoppelverfahrens gelungen ist, sehr hohe Strahlintensitäten durch Glasfasern zu transportieren. Die Charakteristika von Schnitten und Bohrungen an avitalem Meniscusknorpel und Knochen, die wir zum Studium der Gewebetrennung mit Hilfe von fasergeführten Excimerlasem durchführten, sind vergleichbar zu konventionellen chirurgischen Methoden.

Für die Ablation des Gewebes wurde der Excimerlaserstrahl (Wellenlänge $308 \mathrm{~nm}$ ) mittels Quarzglasfasern eines Kerndurchmessers zwischen $400 \mu \mathrm{m}$ und $1000 \mu \mathrm{m}$ und einem 
trichterförmigen Einkoppelstück an den Ort der Intervention geführt. Bei Energiedichten bis zu $18 \mathrm{~J} / \mathrm{cm}^{2}$ konnten Ablationsraten von $3 \mu \mathrm{m} / \mathrm{SchuB}$ erzielt werden. Eine Carbonisation findet nicht stalt, wenn in feuchtem Medium gearbeitet wird; auch bei längerer Bestrahlung erhöht sich die Temperatur in umliegenden Bereichen nur um wenige Grad. Der Nekrosesaum ist nur wenige Mikrometer stark.

Durch Variation der applizierten Energiedichte $\left(0,5 \mathrm{~J} / \mathrm{cm}^{2}\right.$ bis $\left.18 \mathrm{~J} / \mathrm{cm}^{2}\right)$ der verwendeten Repetitionsrate (bis zu $100 \mathrm{~Hz}$ ) und der Pulsdauer ( $25 \mathrm{~ns}, 60 \mathrm{~ns}$ und $300 \mathrm{~ns}$ ) des Excimerlasers konnten optimale Parameter gefunden werden, um damit Schnitte und Bohrungen im Knochen und Knorpel herzustellen, deren Qualität mit der konventioneller Methoden vergleichbar ist. Es sind zur Zeit Schnitte mit einer Breite von $0,5 \mathrm{~mm}$ bis $1,5 \mathrm{~mm}$ möglich. Die Bohrgeschwindigkeit beträgt $2 \mathrm{~mm} / \mathrm{s}$ im Meniscus, und $0,1 \mathrm{~mm} / \mathrm{s}$ im Knochen. Ein Meniscus $(4 \mathrm{~mm} \times 13 \mathrm{~mm})$ ist in ca. $100 \mathrm{~s} z u$ durchtrennen. Das Profil zeigt scharfe Ränder und ist auch bei großer Tiefe ( $>3 \mathrm{~mm}$ ) im wesentlichen rechteckig; nur die Spitze läuft leicht konisch zu. Die Schnittfiächen sind glatt. Diese Resultate werden durch rastcrelektronenmikroskopische und histomorphologische Untersuchungen bestätigt. 